\title{
Radio Forensics Could Unmask Nearby Off-axis Gamma-ray Bursts
}

\author{
I. Bartos ${ }^{\star}{ }^{1}$ K.H. Lee ${ }^{1}$ A. Corsi, ${ }^{2}$ Z. Márka ${ }^{3,4}$ S. Márka ${ }^{3,4}$ \\ ${ }^{1}$ Department of Physics, University of Florida, Gainesville, FL 32611, USA \\ ${ }^{2}$ Department of Physics and Astronomy, Texas Tech University, Lubbock, TX 79409, USA \\ ${ }^{3}$ Columbia Astrophysics Laboratory, Columbia University, New York, NY 10027, USA \\ ${ }^{4}$ Department of Physics, Columbia University, New York, NY 10027, USA
}

12 March 2019

\begin{abstract}
The multi-messenger observation of gamma-ray burst (GRB) 170817A from the nearby binary neutron-star merger GW170817 demonstrated that low-energy $\gamma$-ray emission can be observed at relatively large angles from GRB jet axes. If such structured emission is typical, then the currently known sample of short GRBs with no distance measurements may contain multiple nearby off-axis events whose delayed afterglows could have gone undetected. These nearby neutron star mergers may produce telltale radio flares peaking years after the prompt GRB emission that could still be observable. Here, we show that several short GRBs observed by the Burst Alert Telescope (BAT) on the Neil Gehrels Swift satellite, with no identified afterglow and no distance measurement, could potentially be associated with radio flares detectable by sensitive cm-wavelength radio facilities such as the Karl G. Jansky Very Large Array. We also examine optical follow-up observations that have been carried out for these events, and find that a nearby GW170817-like kilonova is ruled out for only a third of them.
\end{abstract}

Key words: gamma-ray burst: general - radio continuum: transients - gravitational waves

\section{INTRODUCTION}

The remarkable multi-messenger discovery of the binary neutron star (BNS) merger GW170817 by the LIGO/Virgo gravitational-wave (GW) detectors and partner electromagnetic (EM) observatories, has marked the start of a new era in astrophysics. In addition to crowning multi-messenger astrophysics, confirming the predictions of Einstein's general theory of relativity, and clarifying the origin of the heaviest elements of the periodic table, GW170817 has given us new insight into the angular structure of relativistic jets in gamma-ray bursts (GRBs; e.g., Abbott et al. 2017a,c; Coulter et al. 2017; Kilpatrick et al. 2017; Hallinan et al. 2017; Tanaka et al. 2017; Cowperthwaite et al. 2017; Villar et al. 2017; Alexander et al. 2017; Margutti et al. 2017; Troja et al. 2017a; Kasliwal et al. 2017; D'Avanzo et al. 2018; Margutti et al. 2018; Mooley et al. 2018a; Albert et al. 2017; Dobie et al. 2018; Lazzati et al. 2018; Corsi et al. 2018; Metzger 2017a and references therein).

At about $40 \mathrm{Mpc}$ from Earth, GW170817 was associated with the closest and least energetic short GRB we know of (GRB 170817A; Coulter et al. 2017; Goldstein et al. 2017).

^ E-mail: imrebartos@ufl.edu
Its delayed afterglow, first detected in X-rays 9 days after the merger (Troja et al. 2017a; Haggard et al. 2017), and in radio 15 days after the merger (Hallinan et al. 2017), further indicated its unusual nature. These observations suggested that the GRB was off-axis - its jet pointed away from Earth. Continued X-ray/optical/radio follow-up enabled a detailed reconstruction of the properties of the relativistic outflow of GRB 170817A (Alexander et al. 2018; Ruan et al. 2018; Mooley et al. 2018b; Lazzati et al. 2018). Overall, the emerging picture is that GRB 170817 produced structured ejecta with a central, energetic jet core of half opening angle $\theta_{\mathrm{j}} \sim 10^{\circ}$ (Pooley et al. 2018), plus fainter emission that extended to larger angular scales (tens of degrees) from the jet axis. The cause of this structured emission is not yet clear. It could originate from radiation from a very energetic but inefficient (in radiating gamma-rays) cocoon surrounding the ejecta (Mooley et al. 2018a,b; Nakar et al. 2018), or a steep angular structure around the core, where the energy content at large angles is strongly diminished but the efficiency remains high (Lazzati et al. 2017b; van Eerten et al. 2018; Margutti et al. 2017). Structured emission has been previously suggested based on the observed spectral breaks in afterglow emission Rossi et al. (2002) and the observed properties of prompt GRB emission (Eichler \& Levinson 2004). At the

(C) 0000 The Authors 
same time the non-detection of a short GRB brighter than any of the archival events limits the range of opening angles Beniamini et al. (2019a). It is worth noting that similar gamma-ray emission at large angles is likely strongly suppressed in long GRBs, as indicated from the comparison of their prompt and afterglow properties and by considering the shape of their luminosity function (Beniamini \& Nakar 2019).

Prior to GRB 170817A, observations of so-called jet breaks in the X-ray and optical afterglow light curves of several GRBs indicated that these explosions are highly beamed (Mészáros 2006). Typical reconstructed half opening angles for short GRBs are around $\theta_{\mathrm{j}} \sim 10^{\circ}$ (Berger 2014; Fong et al. 2015). Nonetheless, evidence for more structured jets had emerged in some cases (e.g., Perna et al. 2003). Some afterglow observations suggested a two component jet with an inner, narrow ultra-relativistic core, and an outer, wider and mildly relativistic component (Frail et al. 2000; Berger et al. 2003; Sheth et al. 2003). Structured emission was also motivated theoretically (Lipunov et al. 2001; Zhang \& Meszaros 2002; Pescalli et al. 2015; Starling et al. 2005).

The structure of short GRB jets has important consequences for the electromagnetic observability and multimessenger detection rate of BNS mergers. In particular, sufficiently nearby GRBs may be detected in $\gamma$-rays even if their luminous jet core is not beamed towards Earth (offaxis jet), due to the weaker emission from the jet wings (Lazzati et al. 2017a, 2018; Mooley et al. 2018a; Ioka \& Nakamura 2018; Granot et al. 2017). This makes the local BNS merger population over-represented within the detected sample of GRBs. Taking the structured emission of GRB 170817A as standard, Gupte \& Bartos (2018) found that $\sim 10 \%$ of observed short GRBs could be associated with BNS mergers within $200 \mathrm{Mpc}$, although this fraction could be less if GRB 170817A is atypical (Beniamini et al. 2019b). A large fraction of local short GRBs has also been inferred from their directional correlation with nearby galaxies (Tanvir et al. 2005).

Detecting the afterglow of a potential population of nearby, off-axis short GRBs may not be as straightforward as for the case of on-axis events. Indeed, off-axis afterglow emission peaks later than typically expected for on-axis GRBs, and larger viewing angles result in weaker afterglows (Rossi et al. 2002; Kumar \& Granot 2003; Granot \& Kumar 2003; van Eerten et al. 2010; Rossi et al. 2019). Kilonova emission could be more promising as it is isotropic and may be detected for about a week after the merger from nearby events (or even more distant ones if accurate localization enables deep follow-up; Tanvir et al. 2013; Troja et al. 2018a). In fact, the lack of such a kilonova signature in deep followup observations of nearby GRB candidates has been used to constrain the nature of GRB progenitors and/or to set a lower limit on the actual GRB distance under the hypothesis of a binary merger origin (see e.g., Levan et al. 2008, and Section 4).

Before GW170817, short GRBs detected by Swift lacking arcsec localizations have not been monitored systematically for kilonova emission, leaving many of them unconstrained. Thus, the question of whether one could still identify these events as nearby $(\lesssim 200, \mathrm{Mpc})$ long after the GRB explosion, has gained renewed interest. Here, we explore whether radio remnants of BNS mergers may be the
Rosetta Stone that establishes the nearby origin of off-axis GRBs, even long after their observation in $\gamma$-rays. During the merger, NSs eject some of their matter through tidal disruption and winds. The interaction of this mildly-relativistic matter with the circum-merger medium may give rise to radio emission that can last for years (Nakar \& Piran 2011). This very-late-time radio emission could be identified out to possibly $200-300 \mathrm{Mpc}$, depending on the density $n$ of the circum-merger medium (Hotokezaka et al. 2016; Kathirgamaraju et al. 2019). Searches for radio remnants of short GRBs carried out so far have targeted only well localized events, all located at $>500 \mathrm{Mpc}$ (Horesh et al. 2016; Fong et al. 2016). These searches have resulted in non-detections only, and were focused on the search for long-lived magnetar remnants from the binary merger. In this paper we show that, if GRB ejecta are structured similarly to GW170817, then radio observatories such as the Karl G. Jansky Very large Array (VLA) may help us identify nearby events in the sample of previously detected short GRBs with no observed afterglows and unknown distances.

Our discussion is organized as follows. In Section 2 we discuss the expected detection rate of nearby short GRBs and show that there should be some nearby GRBs among those detected so far. In Section 3 we review why these nearby GRBs could have gone unidentified as local events. In Section 4 we introduce Swift/BAT GRBs of interest that have no identified afterglows. We also discuss constraints on their distances from previous optical observations that rule out a nearby kilonova (for some of the bursts). In Section 5 we calculate the expected radio remnant flux for GRBs in our sample, discuss their detectability with the VLA, and their expected time evolution. In Section 4.2 we comment on directionally overlapping nearby galaxies for the same GRBs. We conclude in Section 6.

\section{EXPECTED DETECTION RATE OF NEARBY SHORT GRBs}

For structured jets like GRB 170817A, a GRB can be observed even off-axis - at viewing angles $\theta_{\mathrm{v}}>\theta_{\mathrm{j}}$-as long as the GRB is sufficiently nearby, where the close distance compensates for the weak emission (Lazzati et al. 2017a, 2018; Mooley et al. 2018a; Ioka \& Nakamura 2018; Granot et al. 2017). Observability out to greater $\theta_{\mathrm{v}}$ means a higher rate of detection. Therefore, there should be more observed nearby GRBs than expected from the rate of distant GRBs, and nearby GRBs should typically be observed at greater $\theta_{\mathrm{V}}$ than distant GRBs. The reconstructed large viewing angle of $14^{\circ} \lesssim \theta_{\mathrm{v}} \lesssim 40^{\circ}$ from the nearby GRB $170817 \mathrm{~A}$ is in line with this expectation (Abbott et al. 2018a; Finstad et al. 2018).

If GRB 170817A is typical, then similar, nearby short GRBs with large $\theta_{\mathrm{v}}$ should have been detected in the past. Considering a detection threshold fluence of $2.5 \times 10^{-8} \mathrm{erg} \mathrm{cm}^{-2}$ and GRB 170817A's fluence of $\approx 3 \times$ $10^{-7} \mathrm{erg} \mathrm{cm}^{-2}$, both for Fermi-GBM in the $10-1000 \mathrm{keV}$ energy range (Goldstein et al. 2017), a GRB 170817A-like event could be observed out to a distance of $\sim 130 \mathrm{Mpc}$. This distance grows further for smaller $\theta_{\mathrm{v}}$.

To estimate the expected number of detected nearby short GRBs, Gupte \& Bartos (2018) considered the struc- 
tured jet model of Margutti et al. (2018). They took the local BNS merger rate of $1540_{-1220}^{+3200} \mathrm{Gpc}^{-3} \mathrm{yr}^{-1}$ inferred from LIGO/Virgo observations (Abbott et al. 2017a), and considered that Fermi (Swift) monitors $70 \%$ (15\%) of the sky at any given time. They found that about $10 \%$ of detected short GRBs from BNS mergers should have occurred within $200 \mathrm{Mpc}$, the average distance out to which GW observatories will be able to detect BNS mergers when reaching full sensitivity (Abbott et al. 2018b). Considering that the total short GRB detection rate of Fermi/GBM and Swift/BAT is $40 \mathrm{yr}^{-1}$ (Narayana Bhat et al. 2016) and $8 \mathrm{yr}^{-1}$, respectively, up to tens of previously observed GRBs could be of local origin. Beniamini et al. (2019a) used the observed shortGRB luminosity function to calculate the expected fraction of nearby, off-axis short GRBs. They find a somewhat smaller fraction, $1 \%-10 \%$ for these events, and conclude that GRB 170817 is probably unusual.

\section{COULD NEARBY SHORT GRBs GO UNRECOGNIZED?}

Despite the non-negligible expected rate of nearby $(\lesssim$ $200 \mathrm{Mpc}$ ) short GRBs, none had been identified prior to GW 170817A, and GRB 170817A itself would probably not have been recognized as a nearby event without the GW detection motivating an aggressive broad-band follow-up campaign in its relatively large sky localization area (Abbott et al. 2017c; Coulter et al. 2017). Indeed, $\gamma$-rays alone mostly cannot provide accurate enough localization to allow for host galaxy identification and distance measurement. Detecting GRB afterglows, which could provide arcsec localizations, is only straightforward for on-axis events, for which the X-ray afterglow is the brightest shortly after the GRB, following which it gradually fades (Evans et al. 2009; Nousek et al. 2006). The X-ray afterglow of an off-axis GRB, on the other hand, is weaker at peak compared to an on-axis event, and the peak time is delayed possibly by days after the GRB (van Eerten et al. 2010; van Eerten \& MacFadyen 2011). So far, within the sample of short GRBs, a delayed X-ray afterglow has been detected for only two bursts: GRB 150101B, whose X-ray afterglow was fortuitously identified thanks to its vicinity to a low-luminosity AGN initially mistaken for the candidate X-ray counterpart (Troja et al. 2018b); and GRB 170817A, for which the GW localization led to the identification of the optical kilonova to arcsec position, thus allowing X-ray/radio follow-up.

The optical kilonovae that are produced in the aftermath of BNS mergers by dynamical and wind ejecta last for about a week, with optical/near-infrared emission of $10^{40}-10^{42} \mathrm{erg} \mathrm{s}^{-1}$ (Metzger 2017b; Cowperthwaite et al. 2017). Although kilonovae have the advantage of being potentially observable regardless of viewing geometry, their optical/NIR luminosity makes it challenging for most current large optical surveys to discover them (Yang et al. 2017; Cowperthwaite et al. 2017; Scolnic et al. 2018). This will likely change in the future with the completion of large-scale survey instruments such as the Large Synoptic Survey Telescope (Cowperthwaite et al. 2018). Kilonova emission can be more easily identified if a GRB is detected in association with it, thus enabling more accurate localization (Tanvir et al. 2013). While the arcmin-level localizations available for short GRBs without an observed X-ray afterglow can still challenge the identification of a kilonova at large distances (Singer et al. 2015), a kilonova from nearby GRBs with arcmin localizations could be detected using systematic, targeted follow-up with meter-class telescopes (see Appendix and Table 1).

As discussed in Section 1, slow ejecta from BNS mergers should also produce radio flares by interacting with the surrounding medium. These flares are the focus of this paper. They can last for years after the merger (Nakar \& Piran 2011; Hotokezaka et al. 2016), and could be detectable from nearby $(\lesssim 200 \mathrm{Mpc}$ ) events, depending on the ejecta velocity distribution and the density of the circum-merger medium. However, typically, only short GRBs localized to arcsec positions are extensively followed up in the radio, and all of these well localized short GRBs (other than GRB 170817A) reside beyond $\sim 1 \mathrm{Gpc}$, which makes it unlikely for a radio flare from slow BNS ejecta to be detectable. Hereafter, we explore the possibility of detecting very-late-time radio flares from BNS ejecta associated with nearby short GRBs lacking an X-ray afterglow identification (possibly because off-axis), and localized to arcmin positions only thanks to their $\gamma$-ray emission.

\section{NEARBY GRB CANDIDATES}

To identify promising candidates for nearby GRBs, we considered short GRBs in the Swift catalog with no afterglow detection and thus no known distance measurement. We only considered short GRBs detected by Swift/BAT as they have $90 \%$ error radii smaller than half the FWHM of the VLA primary beam at $6 \mathrm{GHz}(\approx 7.5 \mathrm{arcmin})$, making them suitable to be covered with a single VLA pointing. We excluded from the sample GRBs with Declination below $-40^{\circ}$ as those would not be observable with the VLA, and also GRBs with declinations in the range Dec $=\left[-5^{\circ}, 15^{\circ}\right]$ as observations of them can be significantly degraded due to satellite transmission. We list the remaining, suitable GRBs in Table 1.

\subsection{Constraints from previous observations}

Among other properties, in Table 1 we list the $\gamma$-ray fluence of GRBs in our sample as measured by Swift/BAT. The measured fluences range from $f_{\gamma}=\left(1.2 \times 10^{-8}-2.6 \times\right.$ $10^{-7}$ ) $\mathrm{erg} \mathrm{cm}^{-2}$ in the $15-150 \mathrm{keV}$ band, typical for short GRBs detected by Swift/BAT (Lien et al. 2016). Considering a fiducial distance of $200 \mathrm{Mpc}$, these fluences can be converted to isotropic-equivalent $\gamma$-ray energies as (see Eq. 6 of Fong et al. 2015):

$E_{\gamma, \text { iso }}=k_{\text {bol }} \times \frac{4 \pi d_{\mathrm{L}}^{2}}{1+z} f_{\gamma}$,

where $k_{\text {bol }}=5$ is the bolometric correction factor to convert the fluence to an energy range of $\approx 1-10^{4} \mathrm{keV}, d_{\mathrm{L}}=200 \mathrm{Mpc}$ is the luminosity distance, and $z$ is the redshift. Using this conversion we obtain a range of $E_{\gamma \text {,iso }}=3 \times 10^{47}-6 \times 10^{48} \mathrm{erg}$. For comparison, on axis short GRBs are measured to have $E_{\gamma, \text { iso }}=3 \times 10^{49}-3 \times 10^{52} \mathrm{erg}$ (e.g., Fig 7 of Fong et al. 2015 ), while GRB $170817 \mathrm{~A}$ was measured to have $E_{\gamma \text {,iso }} \approx$ $3 \times 10^{46} \mathrm{erg}$. This implies that it is unlikely that GRBs in 
our sample on-axis bursts located at $d_{L} \lesssim 200 \mathrm{Mpc}$ (see also Beniamini et al. 2019a). However, their fluences are consistent with both nearby but off-axis GRBs, and distant but on-axis events.

We further examined whether optical follow-up observations of these GRBs - which were primarily aimed at identifying their afterglow emission - can set constraints on their distances assuming they are all associated with GW170817like isotropic kilonovae. Specifically, we considered the observed kilonova light curve of GW170817, and checked for each GRB whether the available optical follow-up observations could have uncovered such a kilonova at a distance of $d_{L} \lesssim 200$ Mpc. We found that about a third of the GRBs in our sample are constrained by these observations to be either farther than $200 \mathrm{Mpc}$, or not associated with a GW170817like kilonova. We mark these GRBs in the last column of Table 1 as they are not of high interest for a search of nearby radio flares as described in Section 5 (see the Appendix for further details).

Finally, Table 1 also lists available exclusion distance lower limits from astrophysically triggered searches (Abbott et al. 2008) using GW data. Of the GRBs in our sample, only GRB 151228A has a measured exclusion distance lower limit of $122 \mathrm{Mpc}$ for a BNS merger, which sets a lower distance limit for this event (Abbott et al. 2017b).

\subsection{Spatial correlation with local galaxies}

As an independent check for nearby GRBs in our sample, we searched for spatially coincident galaxies with luminosity distances below $200 \mathrm{Mpc}$ using a publicly available galaxy catalog (Dálya et al. 2018). The catalog has a stated completeness of $40 \%$ in blue luminosity within $200 \mathrm{Mpc}$, therefore a lack of overlap does not rule out the local origin of a GRB.

We searched for galaxies within $200 \mathrm{Mpc}$ in the catalog whose angular distance from the direction of GRBs in our sample is less than the sum of the GRB's $90 \%$ error radius and an offset corresponding to a projected physical offset of $30 \mathrm{kpc}$. The projected offset was calculated for every galaxy individually. This latter term takes into account that BNS systems can move away from their host galaxies due to natal kicks experienced by neutron stars at their formation. We use $30 \mathrm{kpc}$ as it includes $\sim 90 \%$ of observed projected physical offsets for short GRBs (Berger 2014). We found that GRB 050906 has two and spatially coincident galaxies within $200 \mathrm{Mpc}$, at $130 \mathrm{Mpc}$ and $150 \mathrm{Mpc}$ from Earth. While in the sky area contaminated by satellite transmission, we note that GRB 070810B also has a directionally coincident galaxy at $170 \mathrm{Mpc}$ from Earth.

To determine the probability of a chance coincidence, we counted the number of galaxies within $200 \mathrm{Mpc}$ of Earth and within $2^{\circ}$ of the direction of GRBs in our sample, but outside of the $90 \%$ error radius plus $30 \mathrm{kpc}$ offset. We used this number for each GRB to estimate the expected number of chance coincidences. We found that the probability of observing each galaxy as a chance coincidence for GRB 050906 and GRB 070810B is of 9\% and 5\%, respectively. Therefore, these galaxy overlaps by themselves do not establish the local origin of GRB 050906 and GRB 070810B. Moreover, optical non-detections rule out a GW170817-like kilonova at $d_{L} \lesssim 200 \mathrm{Mpc}$ for both these events (see Table 1 ).

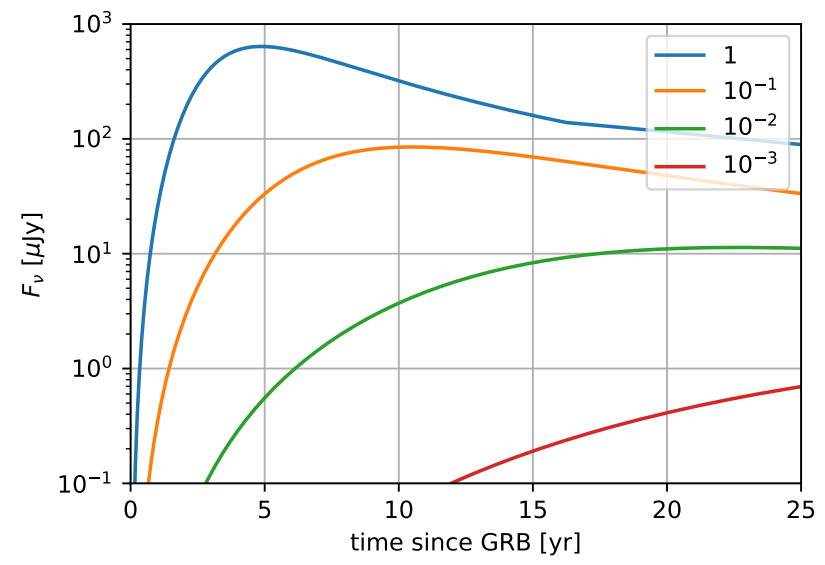

Figure 1. Expected $6 \mathrm{GHz}$ radio flux from a BNS ejecta for a source at $200 \mathrm{Mpc}$ as a function of time after merger, for different circum-merger densities (see legend; densities are in units of $\mathrm{cm}^{-3}$ ). The ejecta was assumed to have a fast component with $0.01 \mathrm{M}_{\odot}$ mass expanding with $0.3 c$ speed, and a slow component with $0.04 \mathrm{M}_{\odot}$ mass expanding with $0.1 c$ speed.

\section{IDENTIFYING NEARBY SHORT GRBs WITH RADIO FORENSICS}

Here, we are interested in testing whether any of the GRBs in our sample could be identified as nearby by means of the very late-time radio flares expected to be associated with BNS slower ejecta. We make the assumptions that all GRBs in our sample produce slow ejecta with properties similar to the slow ejecta that powered the GW17017 kilonova. Under this assumption, we discuss the predictions for the expected luminosity of the associated radio flares (Section 5.1). Then, we present the practical implementation of a search for latetime radio flares with the VLA (Section 5.2).

\subsection{Expected radio emission}

We calculate the radio flare light curve expected for different BNS circum-merger densities. We adopt an outflow compatible with the reconstructed properties of of the kilonova associated with GW170817. These properties indicated the presence of two components: a faster component with $0.01 \mathrm{M}_{\odot}$ mass and $0.3 c$ velocity, and a slower component with $0.04 \mathrm{M}_{\odot}$ mass and $0.1 c$ velocity (Cowperthwaite et al. 2017). We assumed that all BNS ejecta have these two components.

We calculate the expected radio fluxes following the prescriptions of Piran et al. (2013). We consider an expanding spherical, non-relativistic ejecta that collects mass from the uniform circum-merger medium with particle density $n$ as it moves outwards with velocity $v \equiv \beta c$, where $c$ is the speed of light. The velocity of the ejecta decreases as it collects more mass. The relation between ejecta velocity and radius $(R)$ can be calculated by assuming that kinetic energy is conserved (see Eq. 14 of Piran et al. 2013):

$M(R)(\beta c)^{2} \approx E(\geq \beta)$,

where $M$ is the collected mass at radius $R$ from the merger, and $E(\geq \beta)$ is the kinetic energy of the part of the ejecta that was launched with velocities faster than $\beta c$. In our case, this 
means that initially only the component with $0.3 c$ velocity affects the evolution of the ejecta front and hence the radio flux until it slows down to $0.1 c$ where the slower component can catch up. With $R$ and $\beta$ known functions of time, we can calculate the emitted radio flux as (see Eqs. 4 and 6 and Table 2 in Piran et al. 2013)

$F(t) \approx 4$ mJy $R_{17}^{3} n_{-1}^{15 / 8} \beta^{19 / 4} d_{200}^{-2}\left(\frac{v}{6 \mathrm{GHz}}\right)^{-3 / 4}$,

where $R_{17} \equiv\left(R / 10^{17} \mathrm{~cm}\right), n_{-1} \equiv\left(n / 0.1 \mathrm{~cm}^{-3}\right)$, and $d_{200} \equiv$ $(d / 200 \mathrm{Mpc})$. We assume that the observational frequency $v_{\mathrm{obs}}=6 \mathrm{GHz}$ is greater than both the typical synchrotron frequency of electrons in the forward shock driven by the ejecta $\left(v_{\mathrm{m}}\right)$, and the synchrotron self-absorption frequency ( $v_{\mathrm{a}}$; see e.g., Piran et al. 2013). We further assume that electrons and the magnetic field carry $\epsilon_{\mathrm{e}} \approx \epsilon_{\mathrm{m}} \sim 10 \%$ of the total internal energy of the shocked gas, and that the power-law index of the distribution of the accelerated electrons' Lorentz factors is $p=2.5$.

Our estimated radio light curves are shown in Fig. 1 for different circum-merger densities. Our results are similar to those of Piran et al. (2013), who simulated the mass ejection from BNS mergers and found that most mass will be ejected at $\sim 0.3 c$. Our flux is also dominated by the $0.3 c$ component; the slower component only plays a role on longer time scales than the first two decades after the GRB we focus on here. Our results are also similar to those of Kathirgamaraju et al. (2019) for their $0.3 c$ component after scaling the results to the same distance and radio frequency. At the same time, our estimated flux evolution is significantly slower than that of Hotokezaka et al. (2016). This is because Hotokezaka et al. (2016) assumed that much of the ejecta has a velocity higher than $0.3 c$, which results in faster rise time and more rapid fading of the radio remnant.

\subsection{Identification with the VLA}

Based on the predictions described in Section 5.1, we calculate what would be the expected $6 \mathrm{GHz}$ radio flux for observations of the short GRBs in our sample (see Section 4), and estimate the maximum distance to which this radio emission could be detected with VLA.

We assumed an observing campaign where each GRB is observed over two epochs, once during the VLA semester 2019A (May 31, 2019; epoch I), and a second time during semester 2020A (May 31, 2020; epoch II). We chose these VLA observing semesters because they are the closest to the current date where the VLA is not in its most compact D configuration, which would pose challenges for disentangling any radio flare from possible contamination from the host galaxy (e.g., Palliyaguru et al. 2016). In fact, the VLA $\approx 1$ arcsec resolution in $\mathrm{B}$ configuration and at $6 \mathrm{GHz}$ is ideal as at $200 \mathrm{Mpc}$ is corresponds to scale of $\approx 0.89 \mathrm{kpc}$. Thus, such resolution would be enough to disentangle radio emission from a radio flare located at $200 \mathrm{Mpc}$, for offsets from its hosts $\gtrsim 3 \mathrm{kpc}$ (the last is comparable to that measured for GW170817, and closer than $90 \%$ of offsets measured for cosmological short GRBs; Levan et al. 2017). We assume that each observing epoch is $1 \mathrm{hr}$ long, which is enough for the VLA to reach an r.m.s. sensitivity of $\approx 3 \mu \mathrm{Jy}$ at $6 \mathrm{GHz}$ in its B configuration (for a nominal bandwidth of $4 \mathrm{GHz}$ and accounting for RFI effects and calibration overhead).

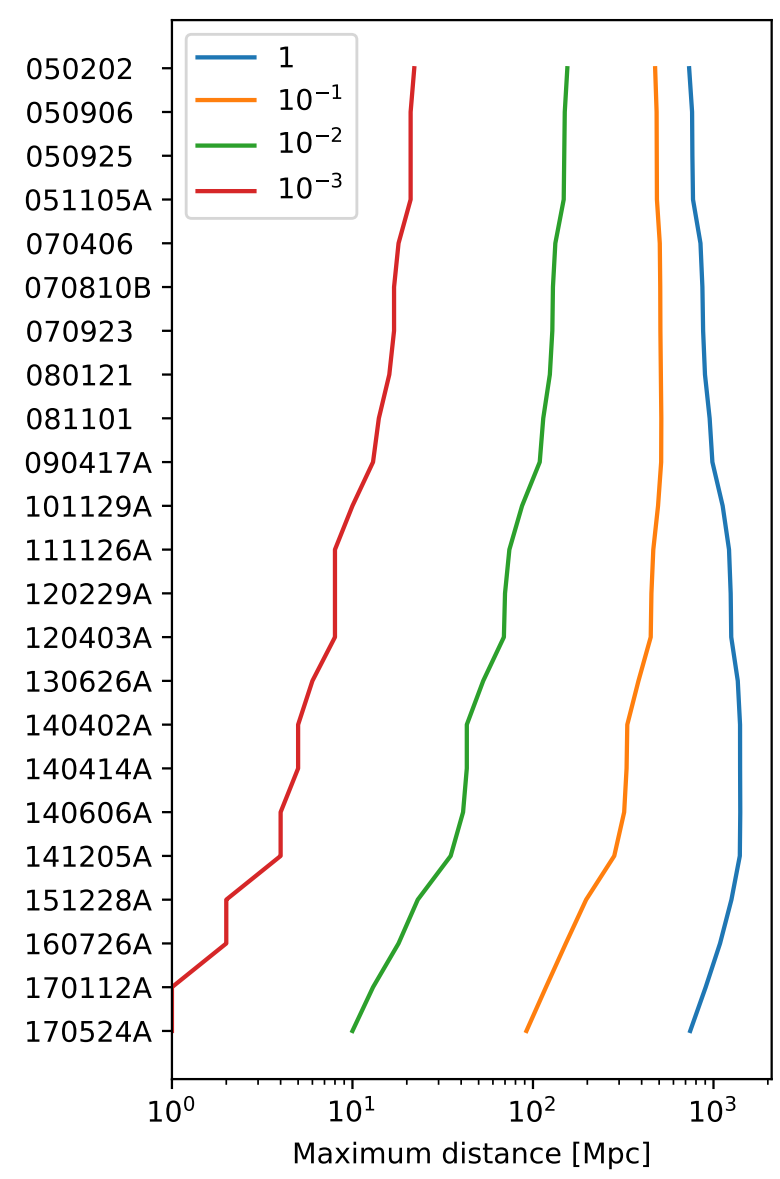

Figure 2. Maximum distance out to which a VLA observation in Epoch I (see Section 5.2) could be used to detect the radio remnant of Swift short GRBs in our sample, for different circummerger medium densities. Swift GRBs used in this analysis are listed along the vertical axis. Circum-merger densities are indicated in the legend, in units of $\mathrm{cm}^{-3}$.

Fig. 2 shows the maximum distances to which radio emission is detectable during Epoch I, assuming that a preGRB radio image is available. For this case, we consider fluxes above $9 \mu \mathrm{Jy}$ to be detectable. For high circum-merger densities, the radio remnant is detected for all GRBs out to large distances. For $1 \mathrm{~cm}^{-3}$ density this is roughly $1 \mathrm{Gpc}$ for all GRBs. However, for circum-merger densities $\lesssim 10^{-3}$, which may represent as much as half of binary mergers (Fong et al. 2015), radio emission is not detectable for our model.

In cases for which no pre-GRB radio images are available, it is also of interest to evaluate whether any radio flux variability can possibly be detected over the two epochs, so it can be used as a discriminant against unrelated radio sources in the field. Specifically, we deem radio variability significant when $\left|F_{\mathrm{I}}-F_{\mathrm{II}}\right| / \sigma_{\left(\mathrm{F}_{\mathrm{I}}-\mathrm{F}_{\mathrm{II}}\right)} \geq 3$, where $\sigma_{\left(\mathrm{F}_{\mathrm{I}}-\mathrm{F}_{\mathrm{II}}\right)}$ is calculated accounting for both the statistical r.m.s. image error, and a 5\% absolute flux calibration error on each of the flux measurements, i.e.:

$\sigma_{\left(\mathrm{F}_{\mathrm{I}}-\mathrm{F}_{\mathrm{II}}\right)} \equiv \sqrt{\left(0.05 F_{\mathrm{I}}\right)^{2}+\left(0.05 F_{\mathrm{II}}\right)^{2}+2 \times(3 \mu \mathrm{Jy})^{2}}$

From Fig. 3 we see that the time variability of the ra- 
Table 1. List of short GRBs detected by Swift/BAT with no identified afterglow and observable with the VLA (see text for discussion). The columns are: GRB name; Right Ascension; Declination; 90\% error radius of the localization; $\gamma$-ray fluence $(15-150 \mathrm{keV})$; delay between the GRB trigger and beginning of Swift/XRT follow-up observation ("-" indicates XRT no observations have been carried out; see more details and references in the Appendix); published $90 \%$ confidence lower limits on the exclusion distance for BNS mergers by targeted GW observations (Abadie et al. 2010, 2012; Abbott et al. 2017b); in the last column we marked GRBs for which optical follow-up observations rule out a GW170817-like kilonova within $200 \mathrm{Mpc}$.

\begin{tabular}{lccccccc}
\hline $\begin{array}{c}\text { GRB } \\
\text { name }\end{array}$ & $\begin{array}{c}\text { R.A. } \\
{[\mathrm{deg}]}\end{array}$ & $\begin{array}{c}\text { Dec. } \\
{[\mathrm{deg}]}\end{array}$ & $\begin{array}{c}\text { err.rad. } \\
{[\mathrm{arcmin}]}\end{array}$ & $\begin{array}{c}f_{\gamma} \\
{\left[\mathrm{erg} \mathrm{cm}{ }^{-2}\right]}\end{array}$ & $t_{\mathrm{XRT}}$ & $\begin{array}{c}D_{\mathrm{GW}} \\
{[\mathrm{Mpc}]}\end{array}$ & $\begin{array}{c}\text { GW170871-like kilonova at } \\
d_{L} \lesssim 200 \mathrm{Mpc} \text { excluded? }\end{array}$ \\
\hline \hline $170524 \mathrm{~A}$ & 319.488 & 48.603 & 2 & $3.7 \times 10^{-8}$ & $61 \mathrm{~s}$ & \\
\hline $170112 \mathrm{~A}$ & 15.232 & 17.233 & 2.5 & $1.3 \times 10^{-8}$ & $62 \mathrm{~s}$ & \\
\hline $160726 \mathrm{~A}$ & 98.809 & -6.617 & 1.3 & $2.6 \times 10^{-7}$ & - & \\
\hline $151228 \mathrm{~A}$ & 214.017 & -17.665 & 1.8 & $8.4 \times 10^{-8}$ & - & 122 \\
\hline $141205 \mathrm{~A}$ & 92.859 & 37.876 & 2 & $1.2 \times 10^{-7}$ & $6.7 \mathrm{~h}$ & \\
\hline $140606 \mathrm{~A}$ & 201.799 & 37.599 & 2.4 & $5.1 \times 10^{-8}$ & $1.3 \mathrm{~h}$ & \\
\hline $140414 \mathrm{~A}$ & 195.31 & 56.902 & 4 & $1.2 \times 10^{-7}$ & $11.2 \mathrm{~h}$ & \\
\hline $130626 \mathrm{~A}$ & 273.128 & -9.525 & 1.8 & $5.2 \times 10^{-8}$ & $111 \mathrm{~s}$ & \\
\hline $120403 \mathrm{~A}$ & 42.458 & 40.489 & 2.3 & $1.0 \times 10^{-7}$ & - & \\
\hline $120229 \mathrm{~A}$ & 20.033 & -35.796 & 1.9 & $4.1 \times 10^{-8}$ & - & \\
\hline $111126 \mathrm{~A}$ & 276.057 & 51.461 & 3 & $7 \times 10^{-8}$ & - & \\
\hline $101129 \mathrm{~A}$ & 155.921 & -17.645 & 3 & $9 \times 10^{-8}$ & $11 \mathrm{~h}$ & \\
\hline $090417 \mathrm{~A}$ & 34.993 & -7.141 & 2.8 & $1.9 \times 10^{-8}$ & - & \\
\hline 080121 & 137.235 & 41.841 & 3 & $3 \times 10^{-8}$ & $2.3 \mathrm{~d}$ & \\
\hline 070923 & 184.623 & -38.294 & 2.1 & $5.0 \times 10^{-8}$ & - & 5.1 \\
\hline 070406 & 198.956 & 16.53 & 3.3 & $4.5 \times 10^{-8}$ & $20.6 \mathrm{~h}$ & \\
\hline $051105 \mathrm{~A}$ & 265.279 & 34.916 & 2.6 & $2.0 \times 10^{-8}$ & $67 \mathrm{~s}$ & \\
\hline 050925 & 303.49 & 34.329 & 1.4 & $7.5 \times 10^{-8}$ & $91 \mathrm{~s}$ & \\
\hline 050906 & 52.802 & -14.621 & 3.3 & $5.9 \times 10^{-8}$ & $79 \mathrm{~s}$ & \\
\hline 050202 & 290.584 & -38.73 & 2.3 & $3.0 \times 10^{-8}$ & - & \\
\hline
\end{tabular}

dio remnants of recent GRBs can be detected to potentially very large distances due to the bright and fast rising radio flare. At later times, while the flare is still bright, its evolution slows down and is therefore the radio remnant's time variability is not detectable unless we have a reference observation from before the GRB.

\section{CONCLUSION}

We examined late-time radio observations to establish whether some of the short GRBs detected in the past by Swift-BAT with no afterglow identification and no distance measurement, could be BNS mergers similar to GW170817 located within $200 \mathrm{Mpc}$.

The detectability of the late-time radio flares associated with slow BNS ejecta depends on several source and environment properties. The most important parameter is the density of the circum-merger medium. Most GRBs in our sample, if observed in 2019 and had a pre-GRB ref- erence image, would have detectable radio flares out to $\gtrsim 500 \mathrm{Mpc}$ for $n \gtrsim 0.1 \mathrm{~cm}^{-3}$, and out to $\gtrsim 100 \mathrm{Mpc}$ for $n \gtrsim 10^{-2} \mathrm{~cm}^{-3}$ (see Fig. 2). Establishing time variability of the radio emission to disentangle the radio counterpart from unrelated sources in the field is more challenging. For this case, we find that variability due to radio flares can be found with our fiducial Epochs I and II observations out to $\sim 200 \mathrm{Mpc}$ for $n \gtrsim 10^{-1} \mathrm{~cm}^{-3}$ (see Fig. 3). For comparison, measured circum-merger densities for cosmological short GRBs with measured afterglow fall in the range of $10^{-4}-1 \mathrm{~cm}^{-3}$ (Fong et al. 2015). The estimated circummerger density of GW170817 is around $5 \times 10^{-3} \mathrm{~cm}^{-3}$ (Lazzati et al. 2018), at which density radio identification is challenging. In this case radio emission for older GRBs could be identified out to the distance of GW170817.

The above estimates depend also on the properties of the kilonova ejecta, in particular its outflow velocity. In our example of two velocity components, the slower, $0.1 c$ has almost no effect on the radio emission in the first 20 years after the merger, even though it carries greater mass. It is 


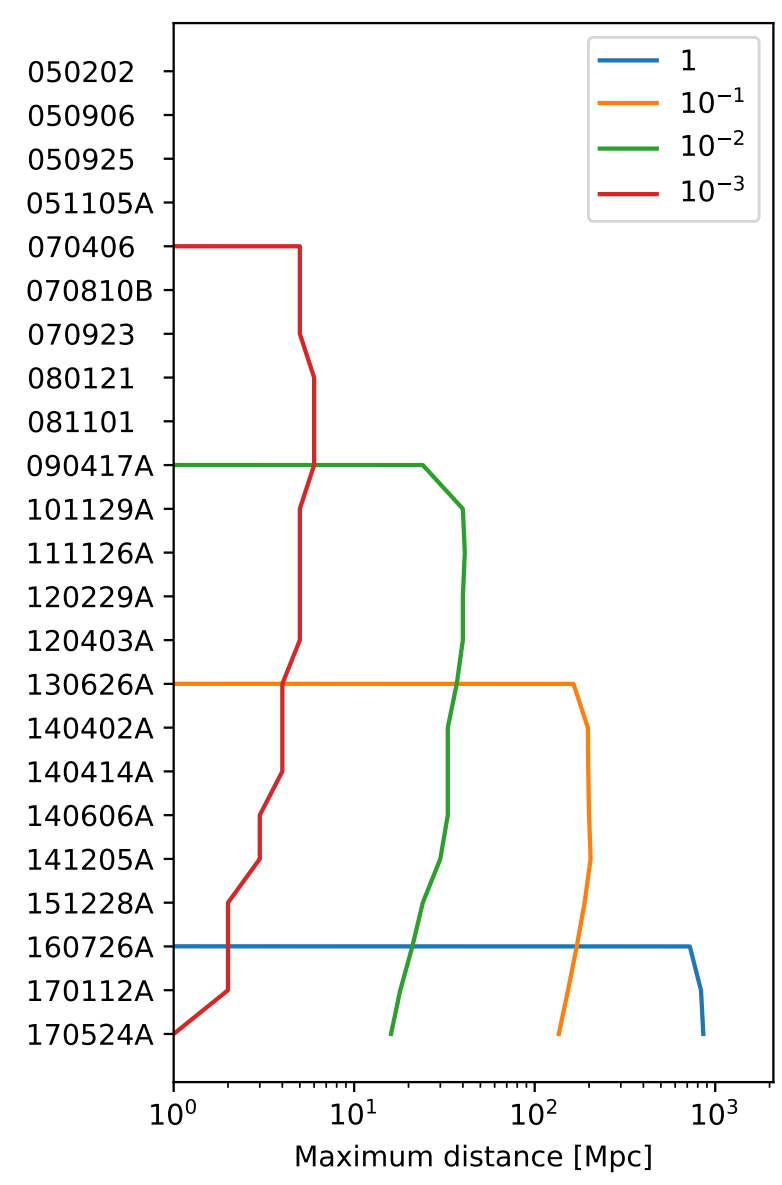

Figure 3. Maximum distance out to which VLA observations in Epochs I and II (see Section 5.2) could be used to assess time variability of the radio remnant of Swift short GRBs in our sample, for different circum-merger medium densities. Swift GRBs used in this analysis are listed along the vertical axis. Circum-merger densities are indicated in the legend, in units of $\mathrm{cm}^{-3}$.

possible that some component of the ejecta reaches velocities greater than our maximum $0.3 c$, which would lead to faster rising and brighter radio emission. For example, Hotokezaka et al. (2016) considers an ejecta velocity distribution that extends to much higher velocities than $0.3 c$. They find that radio emission peaks around $1-6$ years after the merger for $n=1-10^{-3} \mathrm{~cm}^{-3}$, compared to our 5-50 years for the same $n$ range. For such higher velocities, more recent GRBs are more relevant for radio observations, and potentially lower $n$ values can also be probed. It will also be interesting to consider the detectability of long-term X-ray emission from the same interaction between the dynamical/wind ejecta and the circum-merger medium (Beniamini et al. 2018).

The discovery of radio remnants would confirm the BNS origin of a GRB, would provide valuable information on the BNS merger rate, the structure of the relativistic outflows, the properties of kilonova ejecta, and the density of the circum-merger medium. It could also trigger a deep GW search in historical data available for some of the GRBs.

The authors are thankful for the generous support of the University of Florida, Texas Tech University and Columbia University in the City of New York. A.C. acknowledges sup- port from NSF CAREER award 1455090. The Columbia Experimental Gravity group is grateful for the generous support of the National Science Foundation under grant PHY1708028.

\section{APPENDIX: GRB OBSERVATIONS}

Below we summarize the Swift afterglow searches that have been carried out for the short GRBs we consider in this work. We also summarize the optical follow-up that has been carried out, and examine whether the obtained optical upper limits constrain kilonova emission from the source. To this end, we compare the optical constraints to the the observed kilonova light curve of GW170817 from Cowperthwaite et al. (2017) (their Fig. 1; see also Nicholl et al. 2017), shifted from an assumed distance of $40 \mathrm{Mpc}$ to $200 \mathrm{Mpc}$.

- GRB 170524A was detected by Swift/BAT (Gropp et al. 2017). Swift/XRT began observing $60.7 \mathrm{~s}$ after the trigger, and found an uncatalogued X-ray source directionally coincident with the GRB (Gropp et al. 2017). Swift/UVOT began observations $67 \mathrm{~s}$ after the trigger, but found no UV counterpart down to a limiting magnitude of 19.6 mag with its White filter (Gropp et al. 2017; Emery \& Gropp 2017). Several optical telescopes followed up the event, including the $0.5 \mathrm{~m}$ robotic telescope D50 (Trobl et al. 2017), Zeiss1000 (East) of Tien Shan Astronomical Observatory and AZT-8 telescope of CrAO (Mazaeva et al. 2017b), and the Reionization and Transients Infrared Camera (RATIR) on the $1.5 \mathrm{~m}$ Harold Johnson Telescope at the Observatorio Astronomico Nacional (Butler et al. 2017). These observations rule out a GW170817-like kilonova within $1 \mathrm{Gpc}$. For example RATIR constrains the counterpart in the $r$ band to $>25.66 \mathrm{mag} 36$ hours after the burst, at which time GW170817 at $200 \mathrm{Mpc}$ would have had an $\mathrm{r}$ band magnitude of 24.5. Radio follow-up was carried out by the AMI Large Array at $15 \mathrm{GHz}$ beginning 0.2 days, 1.5 days, 3.4 days and 7.4 days after the burst that constrained the source flux density to $162 \mu \mathrm{Jy}, 207 \mu \mathrm{Jy}, 120 \mu \mathrm{Jy}$ and $126 \mu \mathrm{Jy}$ respectively (Mooley et al. 2017).

- GRB 170112A was initially detected by Swift/BAT (Mingo et al. 2017). Swift/XRT began observing $62.3 \mathrm{~s}$ after the trigger, but found no X-ray afterglow (Mingo et al. 2017; D'Ai et al. 2017). Swift/UVOT began observing $66 \mathrm{~s}$ after the trigger, but found no credible optical counterpart but found no UV counterpart down to a limiting magnitude of 19.6 mag with its White filter (Siegel \& Mingo 2017). The event was followed up by optical telescopes, including the 60-cm robotic telescope REM located at the La Silla Observatory (D'Avanzo et al. 2017), the MASTER robotic telescope (R.Podesta et al. 2017), RATIR (Troja et al. 2017b), and the AS-32 $(0.7 \mathrm{~m})$ telescope of Abastumani Observatory (Mazaeva et al. 2017a). The most sensitive of these observations was RATIR's that reached 19 mag in the r, i, Z and Y bands about 3 hours after the burst. While we do not know the luminosity of the GW170817 kilonova at this early time, its peak luminosity expected at $200 \mathrm{Mpc}$ would be about 20.5 mag in these bands. These observations, therefore, cannot rule out a GW170817-like kilonova within $200 \mathrm{Mpc}$. No radio follow-up was reported for this event.

- GRB 160726A was detected by Swift/BAT (Starling et al. 2016), Fermi/GBM (R. Hamburg \& Bissaldi 2016) and 
CALET Gamma-Ray Burst Monitor (Shimizu et al. 2016). Swift/XRT and Swift/UVOT follow-up observations were not carried out due to a Sun observing constraints (Starling et al. 2016). No optical or radio follow-up was reported for this event.

- GRB 151228A was detected by Swift/BAT (Ukwatta et al. 2015) and Fermi/GBM (Elisabetta Bissaldi \& Veres 2015). Swift/XRT and Swift/UVOT follow-up observations were not carried out due to observing constraints (Ukwatta et al. 2015). The 0.6-meter T60 telescope began observing $90 \mathrm{~s}$ after the GRB, and detected no optical afterglow emission (Sonbas et al. 2015). The 0.5-meter MITSuME Akeno optical telescope searched for afterglow emission starting 16.3 hours after the trigger, but detected no emission (Saito et al. 2015). The latter, deeper observation reached a depth of $20 \mathrm{mag}$ in the $\mathrm{R}$ and $\mathrm{g}$ bands, which cannot rule out a GW170817-like kilonova within $200 \mathrm{Mpc}$. No radio follow-up was reported for this event.

- GRB 141205A was detected by Swift/BAT (Cummings 2014c) and Fermi/GBM (Roberts 2014). Swift/XRT and Swift/UVOT began observations 6.65 hours after the trigger, but found no afterglow emission (Starling \& Page 2014; M. M. Chester \& Starling 2014). Swift/UVOT reached a limiting magnitude of 20.7 in the $\mathrm{u}$ band. For comparison, the kilonova from GW170817 at $200 \mathrm{Mpc}$ would have had a u-band flux of 21.7 at 14.5 hours after the merger (based on Evans et al. 2017). We do not expect the kilonova light curve in the $\mathrm{u}$ band to be significantly brighter at 6.65 hours compared to 14.5 hours (e.g., Fig. 5 of Metzger 2017b), therefore we do not consider this as a constraint on kilonovae within $200 \mathrm{Mpc}$. Optical follow-up observations have also been carried out by the $1 \mathrm{~m}$ telescope located at Nanshan, Xinjiang, China, about 9 hours after the merger (Xu et al. 2014b). No counterpart was found down to a limiting magnitude of 20.4 in the $\mathrm{r}$ band. This cannot rule out a GW170817-like kilonova within $200 \mathrm{Mpc}$ that would peak at $21 \mathrm{mag}$ in the $\mathrm{r}$ band. No radio follow-up was reported for this event.

- GRB 140606A was detected by Swift/BAT (Stroh et al. 2014a) Swift/XRT began observing $1.3 \mathrm{hrs}$ after the trigger, but found no X-ray afterglow (Stroh et al. 2014b). Swift/UVOT began observation $68 \mathrm{~s}$ after the trigger, but found no optical afterglow down to a limiting magnitude of 20.8 in the $\mathrm{u}$ band (Marshall \& Stroh 2014). This observation was too early to detect a kilonova signal. Optical follow-up observations have been carried out by several observatories, including the $1 \mathrm{~m}$ telescope located at Nanshan, Xinjiang, China (Xu et al. 2014a), the 0.6m TELMA robotic telescope at the BOOTES-2 astronomical station Malaga, Spain (Jelinek et al. 2014), the AS-32 (0.7m) telescope of Abastumani Observatory (Volnova et al. 2014), and the 6meter BTA (+Scorpio-I) at SAO-RAS, Zelenchuk, Russia (Moskvitin et al. 2014). The deepest observation by BTA 60 hours after the burst limited the r-band flux down to 22 mag. At 60 hours, a GW170817-like kilonova at $200 \mathrm{Mpc}$ would have an r-band flux of 22.3. Therefore we cannot fully rule out a GW170817-like kilonova within $200 \mathrm{Mpc}$. No radio follow-up was reported for this event.

- GRB 140414A was detected by IPN (Cummings 2014a) and Swift/BAT (Cummings 2014a,b). Swift/XRT began observing 11.2 hours after the trigger, but found no Xray afterglow (P. D'Avanzo \& Campana 2014). Swift/UVOT began observation about 11 hours after the trigger, but found no optical afterglow down to a limiting magnitude of 20 with its white filter (F. E. Marshall \& D'Avanzo 2014). This is below the expected flux of $21.7 \mathrm{mag}$ in the narrower u-band of a GW170817-like kilonova at $200 \mathrm{Mpc}$ at 14.5 hours. Optical follow-up observations have been carried out by the DOLORES camera on the 3.6-m TNG Telescope at Canary Islands 15.2 hours after the burst, but found no optical counterpart down to a limiting magnitude of $\sim 22$ in the r band (D'Avanzo et al. 2014). At 15.2 hours, a GW170817like kilonova at $200 \mathrm{Mpc}$ would have an r-band flux of 20.8 . Therefore, these observations rule out a GW170817-like kilonova within $200 \mathrm{Mpc}$.

- GRB 130626A was detected by Swift/BAT (Pasquale 2013). Swift/XRT began observing 111 seconds after the trigger. It found a weak X-ray source, however, this source showed no sign of fading not fade, therefore Swift/XRT data shows no sign of an afterglow (Pasquale 2013; K. L. Page \& Pasquale 2013; Page \& Pasquale 2013). Swift/UVOT began observation 67 seconds after the trigger, but found no credible optical afterglow (Pasquale 2013; K. L. Page \& Pasquale 2013). Such early Swift/UVOT observations do not constrain kilonova emission. Optical follow-up observations have been carried out by RATIR 200 seconds after the GRB (Butler 2013), and the 0.76-m Katzman Automatic Imaging Telescope (KAIT), located at Lick Observatory $97 \mathrm{~s}$ after the GRB (Zheng et al. 2013). No optical counterpart was found. These observations are too early for kilonova detection, therefore they cannot rule out a GW170817-like kilonova. No radio follow-up was reported for this event.

- GRB 120403A was detected by Swift/BAT (Beardmore et al. 2012). Due to a Sun observing constraint, Swift/XRT, and Swift/UVOT could not search for afterglow emission from this event (Beardmore et al. 2012). The MASTER II robotic telescope began observing 9.8 hours after the trigger, but found no optical afterglow down to a limiting magnitude of 16 (Yurkov et al. 2012). The depth of this observation is not sufficient to rule out a GW170817like kilonova at $200 \mathrm{Mpc}$. No radio follow-up was reported for this event.

- GRB 120229A was detected by Swift/BAT (Mangano et al. 2012). Due to a Sun observing constraint, Swift/XRT, and Swift/UVOT could not search for afterglow emission from this event (Mangano et al. 2012). The Magellan/Clay 6.5-meter telescope carried out observations at 9.6 and 33.6 hours after the burst, but found no optical counterpart (Fong et al. 2012). They report a limiting magnitude of 22.4 in the r-band at 9.6 hours after the burst. While no r-band observations of GW170817 were reported at this time, extrapolating later observations to this time from Cowperthwaite et al. (2017) indicate an expected r-band magnitude of 21.2 at $200 \mathrm{Mpc}$. Therefore these observations are sufficient to rule out a GW170817-like kilonova at $200 \mathrm{Mpc}$.

- GRB 111126A was detected by Swift/BAT (Cummings \& Palmer 2011). No follow up observation was carried out by Swift or others as the location of the event was about 29 degrees from the Sun (Cummings \& Palmer 2011).

- GRB 101129A was detected by IPN, Swift/BAT (Cummings et al. 2010), Konus-Wind (Golenetskii et al. 2010), and Suzaku WAM (Ohmori et al. 2010). Swift/XRT and Swift/UVOT began observing 11 hours after the trigger, but found no X-ray or optical afterglow emission (Cum- 
mings et al. 2010). No limiting magnitude was reported for Swift/UVOT. No further optical follow-up was reported.

- GRB 090417A was detected by Swift/BAT (Mangano et al. 2009). Due to an observing constraint, no Swift/XRT nor Swift/UVOT observations were carried out (Mangano et al. 2009). A potential host galaxy was identified in the 2MASS catalog that was within the error region of the GRB, with its angular distance suggestive at roughly $97 \%$ confidence (Fox 2009). The galaxy has a distance of about 400 Mpc (O'Brien \& Tanvir 2009). No optical follow-up observation was reported. VLA carried out follow-up observations at $8.46 \mathrm{GHz}$ within a day of the GRB in the direction of the 2MASS galaxy, but found no counterpart down to a limiting flux of about $100 \mu \mathrm{Jy}$ Chandra et al. (2009).

- GRB 080121 was detected by Swift/BAT (Cummings \& Palmer 2008). Swift/XRT and Swift/UVOT began observing 2.3 days after the trigger, but found no X-ray or optical afterglow emission (Troja \& Burrows 2008; A. Cucchiara 2008). Swift/UVOT reported a limiting magnitude of 20.73 in the v band. For comparison, at 2.3 days, a GW170817-like kilonova at $200 \mathrm{Mpc}$ would have a flux of $22.1 \mathrm{mag}$ in the rband, which should be brighter than the $\mathrm{v}$ band. Two nearby SDSS galaxies were identified within Swift/BAT error radius at a distance of $\approx 200 \mathrm{Mpc}$ (D. A. Perley \& Bloom 2008). Several other, fainter sources were also found to be present in the field, suggesting that the GRB may have occurred in a group or small cluster at this distance. No further optical or radio follow-up was reported.

- GRB 070923 was detected by Swift/BAT (Stroh et al. 2007). Swift/XRT and Swift/UVOT data is not available due to the GRB's direction having been too close to that of the Sun (Stroh et al. 2007). VLA searched for but found no afterglow at a frequency of $8.46 \mathrm{GHz} 5$ days after the event, reaching a limiting flux density of $90 \mu \mathrm{Jy}$ (Chandra \& Frail 2007).

- GRB 070810B was detected by Swift/BAT (Marshall et al. 2007). Swift/XRT and Swift/UVOT began observing 60 seconds after the trigger, but found no X-ray or optical afterglow emission (Marshall et al. 2007; R. Starling \& Marshall 2007a; Brown \& Marshall 2007), although Swift/XRT reported two low-significance X-ray sources (R. Starling \& Marshall 2007a). A second observing epoch with Swift/XRT starting at 42 days detected an X-ray source within the Swift/BAT error circle (R. Starling \& Marshall 2007b). It was not reported whether this source faded with time. Swift/UVOT observations were too early to limit kilonova emission. Optical follow-up observations have been carried out by the KANATA $1.5-\mathrm{m}$ telescope at Higashi-Hiroshima Observatory, Japan 3 minutes after the burst (Uemura et al. 2007), the Xinglong TNT $80 \mathrm{~cm}$ telescope $550 \mathrm{~s}$ after the burst (Xin et al. 2007), the 2-m Faulkes Telescope South 3 minutes after the burst (Guidorzi et al. 2007), the Shajn 2.6m telescope of CrAO (V. Rumyantsev \& Pozanenko 2007) and the Keck I telescope 13 hours and 6 days after the burst (Kocevski et al. 2007). The Shajn telescope observed a marginally detectable object, while others reported no observation. Using the deepest limit from Keck, assuming that kilonova emission at 6 hours is comparable to that at 12 hours after the burst, the obtained limit of $25.5 \mathrm{mag}$ in the r band rules out a GW170817-like kilonova within $200 \mathrm{Mpc}$. This galaxy is within the declination range within which ob- servations are significantly degraded due to satellite transmission, therefore we do not include this GRB in Table 1.

- GRB 070406 was detected by Swift/BAT (Cummings 2007). Swift/XRT and Swift/UVOT began observing 20.6 hours after the trigger (E. Troja \& McBreen 2007; W. Landsman \& McBreen 2007), but found no X-ray or optical counterpart (E. Troja \& McBreen 2007; E. Berger \& Ofek 2007; Butler \& Bloom 2007). Swift/UVOT excluded a counterpart down to a limiting magnitude 22.4 in the u band. For comparison, at 20.6 hours, a GW170817-like kilonova at $200 \mathrm{Mpc}$ would have a flux of $21.5 \mathrm{mag}$ in the u-band. Therefore, the Swift/UVOT observation rules out a GW170817-like kilonova within $200 \mathrm{Mpc}$.

- GRB 051105A was detected by Swift/BAT (Mineo et al. 2005a). Swift/XRT and Swift/UVOT began observing 67 seconds after the trigger, but found no X-ray or optical afterglow emission (Mineo et al. 2005a,b; Brown et al. 2005). The Swift/UVOT observation was too early to constrain kilonova emission. Optical follow-up has been carried out by the 14 inch Automated Response Telescope 2.8 hours after the burst (Torii 2005), the Tautenburg 1.34-m Schmidt telescope 10 hours and 34 hours after the burst (S. Klose \& Stecklum 2005; Klose et al. 2005), TNG equipped with DOLORES 13 hours after the burst (Piranomonte et al. 2005), the MDM $2.4 \mathrm{~m}$ telescope 20 hours after the burst (Halpern et al. 2005), the $1.0 \mathrm{~m}$ Nainital telescope starting 8 hours after the burst (de Ugarte Postigo et al. 2005), the $0.25 \mathrm{~m}$ GETS telescope in the Gunma Astronomical Observatory 3 hours after the burst (Kinugasa \& Torii 2005), and the $1.5 \mathrm{~m}$ telescope of Maidanak Astronomical Observatory 7.6 hours after the burst (Sharapov et al. 2005). No plausible optical counterpart was reported. While for several of these observations no upper limit was reported, based on the magnitudes of the reported objects within the field of view, MDM seems to have had a depth down to $\sim 23 \mathrm{mag}$ in the $\mathrm{r}$ band. At 20.6 hours post-burst when the MDM observation was carried out, a GW170817-like kilonova at $200 \mathrm{Mpc}$ should have an r-band magnitude of 20.8. Therefore, the MDM limits likely rule out a GW170817-like kilonova at $200 \mathrm{Mpc}$.

- GRB 050925 was detected by Swift/BAT (Holland et al. 2005). Swift/XRT and Swift/UVOT began observing 91 and 92 seconds after the trigger, respectively, but found no X-ray or optical afterglow emission (Holland et al. 2005; Rosen et al. 2005; Beardmore et al. 2005). The Swift/UVOT observation was too early to constrain kilonova emission. Optical follow-up has been carried out by the 2-m Faulkes North Telescope 3.3 minutes after the burst (Guidorzi et al. 2005), the 0.8-m telescope at XingLong Observatory, China 2.2 hours after the burst (Qiu et al. 2005), the PAIRITEL $1.3 \mathrm{~m}$ telescope 18 hours after the burst (Bloom 2005). No optical counterpart was found. The first two of these follow-ups were too early to constrain kilonova emission. The PAIRITEL observation reached a limiting magnitude of $\approx 18.5$ in the $\mathrm{K}$ and $\mathrm{H}$ bands, below the expected $\approx 21.5 \mathrm{mag}$ in these bands for a GW170817-like kilonova emission from $200 \mathrm{Mpc}$.

- GRB 050906 was detected by Swift/BAT (Krimm et al. 2005). Swift/XRT began observing 79 seconds after the trigger, but found no X-ray or optical afterglow emission (Krimm et al. 2005; Pagani et al. 2005). Swift/UVOT was in safe mode and could not observe the source (Krimm et al. 2005). Optical follow-ups have been carried out by the Robotic Palomar 60-Inch Telescope 114 seconds after the 
burst (D. B. Fox \& Schmidt 2005), the AEOS Burst Camera $(\mathrm{ABC})$, attached to the AEOS telescope 15 minutes after the burst (H. F. Swan 2005), FORS2 on the 8.2-m Antu Telescope at ESO/Paranal about a day after the burst (and 2005), and the Russian-Turkish 1.5-m telescope 13 hours after the burst (Zhuchkov et al. 2005). No optical counterpart was found. VLT reached a limiting magnitude of 24.5 in the $\mathrm{r}$ band, compared to the 20.8 mag expected from a GW170817-like kilonova at $200 \mathrm{Mpc}$. Therefore, VLT observations rule out a GW170817-like kilonova within $200 \mathrm{Mpc}$.

- GRB 050202 was detected by Swift/BAT (Tueller et al. 2005). No Swift/XRT or Swift/UVOT observations have been reported. The Swope 40-inch telescope at Las Campanas Observatory began observations 6 hours after the trigger, but found no optical afterglow emission down to a limiting magnitude of 16 in the $r$ band (Berger \& Krzeminski 2005). The $0.6 \mathrm{~m}$ telescope at Mt. John University Observatory began observations 12.6 hours after the trigger, but found no optical counterpart down to a limiting magnitude of about 20 in the $\mathrm{r}$ band (Castro-Tirado et al. 2005). For comparison, the $\mathrm{r}$ band magnitude of a GW170817-like kilonova at $200 \mathrm{Mpc} 12$ hours after the merger is 21 . The VLA carrier out two epochs of radio observations but found no radio afterglow emission (Frail \& Soderberg 2005a,b).

\section{REFERENCES}

A. Cucchiara P. S., 2008, GCN, 7217

Abadie J., et al., 2010, ApJ, 715, 1453

Abadie J., et al., 2012, ApJ, 760, 12

Abbott B., et al., 2008, Class. Quantum Grav, 25, 114051

Abbott B., et al., 2017a, Phys. Rev. Lett., 119, 161101

Abbott B. P., et al., 2017b, ApJ, 841, 89

Abbott B., et al., 2017c, ApJL, 848, L12

Abbott B., et al., 2018a, arXiv:1805.11579,

Abbott B. P., et al., 2018b, Living Rev. Relativ, 21, 3

Albert A., et al., 2017, ApJL, 850, L35

Alexander K. D., et al., 2017, ApJL, 848, L21

Alexander K. D., et al., 2018, ApJL, 863, L18

Beardmore A. P., et al., 2005, GCN, 4043

Beardmore A. P., et al., 2012, GCN, 13191

Beniamini P., Nakar E., 2019, MNRAS, 482, 5430

Beniamini P., et al., 2018, MNRAS, 476, 5621

Beniamini P., et al., 2019a, MNRAS, 483, 840

Beniamini P., et al., 2019b, MNRAS, 483, 840

Berger E., 2014, ARA\&A, 52, 43

Berger E., Krzeminski W., 2005, GCN, 3006

Berger E., et al., 2003, Nature, 426, 154

Bloom J. S., 2005, GCN, 4042

Brown P. J., Marshall F. E., 2007, GCN, 6755

Brown P. J., et al., 2005, GCN, 4200

Butler N., 2013, GCN, 14943

Butler N. R., Bloom J. S., 2007, GCN, 6263

Butler N., et al., 2017, GCN, 21152

Castro-Tirado A. J., et al., 2005, GCN, 3018

Chandra P., Frail D. A., 2007, GCN, 6831

Chandra P., et al., 2009, GCN, 9160

Corsi A., et al., 2018, ApJL, 861, L10

Coulter D. A., et al., 2017, Science, 358, 1556

Cowperthwaite P. S., et al., 2017, ApJ. Lett., 848, L17

Cowperthwaite P. S., et al., 2018, arXiv:1811.03098,

Cummings J. R., 2007, GCN, 6247

Cummings J. R., 2014a, GCN, 16110

Cummings J. R., 2014b, GCN, 16111
Cummings J. R., 2014c, GCN, 17137

Cummings J. R., Palmer D. M., 2008, GCN, 7209

Cummings J. R., Palmer D. M., 2011, GCN, 12599

Cummings J. R., et al., 2010, GCN, 11436

D. A. Perley R. J. F., Bloom J. S., 2008, GCN, 7210

D. B. Fox S. B. C., Schmidt B. P., 2005, GCN, 3931

D'Ai A., et al., 2017, GCN, 20440

D'Avanzo P., et al., 2014, GCN, 16112

D'Avanzo P., et al., 2017, GCN, 20438

D'Avanzo P., et al., 2018, A\&A, 613, L1

Dálya G., et al., 2018, MNRAS, 479, 2374

Dobie D., et al., 2018, ApJL, 858, L15

E. Berger D. B. F., Ofek E., 2007, GCN, 6262

E. Troja K. P., McBreen S., 2007, GCN, 6255

Eichler D., Levinson A., 2004, ApJL, 614, L13

Elisabetta Bissaldi B. Z., Veres P., 2015, GCN, 18736

Emery S., Gropp J., 2017, GCN, 21141

Evans P. A., et al., 2009, MNRAS, 397, 1177

Evans P. A., et al., 2017, Science, 358, 1565

F. E. Marshall L. H., D'Avanzo P., 2014, GCN, 16114

Finstad D., et al., 2018, ApJL, 860, L2

Fong W., et al., 2012, GCN, 13084

Fong W., et al., 2015, ApJ, 815, 102

Fong W., et al., 2016, ApJ, 831, 141

Fox D. B., 2009, GCN, 9134

Frail D. A., Soderberg A. M., 2005a, GCN, 3007

Frail D. A., Soderberg A. M., 2005b, GCN, 3009

Frail D. A., et al., 2000, ApJL, 538, L129

Goldstein A., et al., 2017, ApJL, 848, L14

Golenetskii S., et al., 2010, GCN, 11439

Granot J., Kumar P., 2003, ApJ, 591, 1086

Granot J., Guetta D., Gill R., 2017, ApJL, 850, L24

Gropp J., et al., 2017, GCN, 21135

Guidorzi C., et al., 2005, GCN, 4035

Guidorzi C., et al., 2007, GCN, 6758

Gupte N., Bartos I., 2018, arXiv:1808.06238,

H. F. Swan I. S., 2005, GCN, 3936

Haggard D., et al., 2017, ApJL, 848, L25

Hallinan G., et al., 2017, Science, 358, 1579

Halpern J. P., et al., 2005, GCN, 4202

Holland S. T., et al., 2005, GCN, 4034

Horesh A., et al., 2016, ApJL, 819, L22

Hotokezaka K., et al., 2016, ApJ, 831, 190

Ioka K., Nakamura T., 2018, Progr. Theor. Exp. Phys, 2018, 043E02

Jelinek M., et al., 2014, GCN, 16361

K. L. Page S. R. O., Pasquale M. D., 2013, GCN, 14937

Kasliwal M. M., et al., 2017, Science, 358, 1559

Kathirgamaraju A., Giannios D., Beniamini P., 2019, arXiv:1901.00868,

Kilpatrick C. D., et al., 2017, Science, 358, 1583

Kinugasa K., Torii K., 2005, GCN, 4242

Klose S., et al., 2005, GCN, 4203

Kocevski D., et al., 2007, GCN, 6771

Krimm H., et al., 2005, GCN, 3926

Kumar P., Granot J., 2003, ApJ, 591, 1075

Lazzati D., et al., 2017a, MNRAS, 471, 1652

Lazzati D., et al., 2017b, ApJL, 848, L6

Lazzati D., et al., 2018, Phys. Rev. Lett., 120, 241103

Levan A. J., et al., 2008, MNRAS, 384, 541

Levan A. J., et al., 2017, ApJL, 848, L28

Lien A., et al., 2016, ApJ, 829, 7

Lipunov V. M., Postnov K. A., Prokhorov M. E., 2001, Astron. Rep, 45, 236

M. M. Chester F. E. M., Starling R. L. C., 2014, GCN, 17144

Mangano V., et al., 2009, GCN, 9133

Mangano V., et al., 2012, GCN, 12997

Margutti R., et al., 2017, ApJL, 848, L20 
Margutti R., et al., 2018, ApJL, 856, L18

Marshall F. E., Stroh M. C., 2014, GCN, 16358

Marshall F. E., et al., 2007, GCN, 6743

Mazaeva E., et al., 2017a, GCN, 20472

Mazaeva E., et al., 2017b, GCN, 21145

Mészáros P., 2006, Rep. Prog. Phys., 69, 2259

Metzger B. D., 2017a, arXiv:1710.05931,

Metzger B. D., 2017b, Living Rev. Relativ, 20, 3

Mineo T., et al., 2005a, GCN, 4188

Mineo T., et al., 2005b, GCN, 4195

Mingo B., et al., 2017, GCN, 20436

Mooley K. P., et al., 2017, GCN, 21212

Mooley K. P., et al., 2018a, Nature, 554, 207

Mooley K. P., et al., 2018b, Nature, 561, 355

Moskvitin A. S., et al., 2014, GCN, 16411

Nakar E., Piran T., 2011, Nature, 478, 82

Nakar E., et al., 2018, ApJ, 867, 18

Narayana Bhat P., et al., 2016, ApJS, 223, 28

Nicholl M., et al., 2017, ApJL, 848, L18

Nousek J. A., et al., 2006, ApJ, 642, 389

O'Brien P. T., Tanvir N. R., 2009, GCN, 9136

Ohmori N., et al., 2010, GCN, 11443

P. D'Avanzo M. G. B., Campana S., 2014, GCN, 16113

Pagani C., et al., 2005, GCN, 3934

Page K. L., Pasquale M. D., 2013, GCN, 15004

Palliyaguru N. T., et al., 2016, ApJL, 829, L28

Pasquale M. D., 2013, GCN, 14931

Perna R., Sari R., Frail D., 2003, ApJ, 594, 379

Pescalli A., et al., 2015, MNRAS, 447, 1911

Piran T., Nakar E., Rosswog S., 2013, MNRAS, 430, 2121

Piranomonte S., et al., 2005, GCN, 4201

Pooley D., et al., 2018, ApJL, 859, L23

Qiu Y., et al., 2005, GCN, 4036

R. Hamburg E. B., Bissaldi E., 2016, GCN, 19732

R. Starling C. M., Marshall F. E., 2007a, GCN, 6754

R. Starling J. P. Osborne K. L. P., Marshall F. E., 2007b, GCN, 6852

R.Podesta et al., 2017, GCN, 20439

Roberts O. J., 2014, GCN, 17143

Rosen S., et al., 2005, GCN, 4038

Rossi E., Lazzati D., Rees M. J., 2002, MNRAS, 332, 945

Rossi A., et al., 2019, arXiv:1901.05792,

Ruan J. J., et al., 2018, ApJL, 853, L4

S. Klose U. L., Stecklum B., 2005, GCN, 4196

Saito Y., et al., 2015, GCN, 18747

Scolnic D., et al., 2018, ApJL, 852, L3

Sharapov D., et al., 2005, GCN, 4349

Sheth K., et al., 2003, ApJL, 595, L33

Shimizu Y., et al., 2016, GCN, 19751

Siegel M. H., Mingo B., 2017, GCN, 20451

Singer L. P., et al., 2015, ApJ, 806, 52

Sonbas E., et al., 2015, GCN, 18746

Starling R. L. C., Page K. L., 2014, GCN, 17141

Starling R. L. C., et al., 2005, MNRAS, 360, 305

Starling R. L. C., et al., 2016, GCN, 19731

Stroh M. C., et al., 2007, GCN, 6818

Stroh M. C., et al., 2014a, GCN, 16353

Stroh M. C., et al., 2014b, GCN, 16357

Tanaka M., et al., 2017, PASJ, 69, 102

Tanvir N. R., et al., 2005, Nature, 438, 991

Tanvir N. R., et al., 2013, Nature, 500, 547

Torii K., 2005, GCN, 4189

Trobl J., et al., 2017, GCN, 21140

Troja E., Burrows D. N., 2008, GCN, 7224

Troja E., et al., 2017a, Nature, 551, 71

Troja E., et al., 2017b, GCN, 20444

Troja E., et al., 2018a, Nature Communications, 9, 4089

Troja E., et al., 2018b, Nature Commun., 9, 4089
Tueller J., et al., 2005, GCN, 3005

Uemura M., et al., 2007, GCN, 6746

Ukwatta T. N., et al., 2015, GCN, 18731

V. Rumyantsev V., Pozanenko A., 2007, GCN, 6762

Villar V. A., et al., 2017, ApJL, 851, L21

Volnova A., et al., 2014, GCN, 16371

W. Landsman M. C., McBreen S., 2007, GCN, 6258

Xin L. P., et al., 2007, GCN, 6747

Xu D., et al., 2014a, GCN, 16359

Xu D., et al., 2014b, GCN, 17142

Yang S., et al., 2017, ApJL, 851, L48

Yurkov V., et al., 2012, GCN, 13197

Zhang B., Meszaros P., 2002, ApJ, 571, 876

Zheng W., et al., 2013, GCN, 14944

Zhuchkov R., et al., 2005, GCN, 3955

and P. J., 2005, GCN, 3940

de Ugarte Postigo A., et al., 2005, GCN, 4204

van Eerten H. J., MacFadyen A. I., 2011, ApJL, 733, L37

van Eerten H., Zhang W., MacFadyen A., 2010, ApJ, 722, 235

van Eerten E. T. H., et al., 2018, arXiv:1808.06617, 